\title{
Control Size Distribution of Hollow Silica Nanoparticles by Viscosity of Emulsion Template
}

Yuki Nakashima, Chika Takai, Chen Wanghui, Hadi Razavi-Khosroshahi, Takashi Shirai, Masayoshi Fuji*

Advanced Ceramics Research Center, Nagoya Institute of Technology 3-101-1, Honmachi, Tajimi, Gifu 507-0033, Japan

*fuji@ nitech.ac.jp

FAX: +81-572-24-8109 TEL: +81-572-24-8110

\begin{abstract}
This paper describes the effect of the viscosity of the template on the sol-gel synthesis which is useful to rapidly form the shell structure of hollow silica nanoparticles (HSNPs) and control the HSNP sizes. The Poly acrylic acid/ammonia aqueous solution $\left(\mathrm{PAA} / \mathrm{NH}_{3}\right)$ acts as the templates for the silica coating performs in ethanol, which are subsequently removed by water to obtain the HSNPs. The influence of the template viscosity on the silica coating and the morphology of the HSNPs were studied using four kinds of PAAs with different molecular weights (MWs), such as $5,000,25,000,50,000$ and 250,000 . It was found that the viscosity of the $\mathrm{PAA} / \mathrm{NH}_{3}$ templates increased with the increasing MW PAA. Unfortunately, the highest molecular weight of PAA $(250,000)$ used in this study hardly formed templates because of its highly excessive viscosity. The ultra-small HSNPs could be successfully synthesized using PAA 50,000 $_{\text {as }}$ ase template along with a high TEOS conversion ratio (95\%) and a shorter reaction time (2 hours). Moreover, the decreasing MW PAA provided a large size HSNP with a low TEOS conversion.
\end{abstract}

Keywords

Hollow silica nanoparticles

Emulsion template

Molecular weight

Viscosity 


\section{Introduction}

The structure of hollow silica nanoparticles (HSNPs) can be described as a nano-sized cavity surrounded by a thin silica shell. With this special structure, HSNPs have a higher specific surface area [1,2], lower bulk density and lower thermal conductivity [3] than solid silica particles, as well as showing unique light scattering properties [4, 5], loading-releasing behavior [6-9], etc. A number of synthetic routes for the hollow HSNPs has been reported, which were categorized into template and non-template methods. In the non-template method, there are the electrostatic atomization method [10] and spray pyrolysis method [11]. It can mass produce HSNPs, however, the obtained HSNPs are poly-dispersed. In the template method, silica was first coated on the templates to form the silica shells, followed by removing the templates via thermal or chemical treatments [12-14].

In the template method, polymeric templates were commonly used as the conventional template methods $[15,16]$. Although using these templates could promise to obtain hollow structures, the corresponding process for producing HSNPs was not very environmentally benign. In the template removing step, $\mathrm{CO}_{2}$ gas or an organic solution would be generated as byproducts. As a highlight, $\mathrm{Yu}$ et al. [17] successfully obtained HSNPs by using the template of a polyacrylic acid/ammonia aqueous solution ( $\mathrm{PAA} / \mathrm{NH}_{3}$ ) emulsion, which could be easily removed by washing with water after the silica coating. In that study, PAA was first dissolved in the ammonia solution, then the mixture was added to ethanol while stirring to form the emulsion templates. The technique for the silica coating was the Stöber process [18], which was directly performed in the ethanol dispersion of the PAA/ $\mathrm{NH}_{3}$ emulsion templates. This method is useful as not only templates for the HSNPs, but also functional particle containers. $\mathrm{He}$ et al. [19] successfully obtained noble-metal nanoparticles@HSNPs by using the PAA/ $\mathrm{NH}_{3}$ emulsions template. In this study, the PAA had dual roles of a template for the HSNPs and as a captor of the metal source in the HSNPs. However, despite the significant advantages of the soft template method, the much higher mobility of the emulsion templates with respect to polymeric templates led to a required long reaction time (10h) for the formation of the silica shells.

Recent work [20-24] further developed the $\mathrm{PAA} / \mathrm{NH}_{3}$ emulsion template method for better controlling the particle size and shell thickness of the produced HSNPs, as well as shortening the processing time. Particularly, it has been suggested by Takai et al. [24] that the silica coating in the PAA/ $\mathrm{NH}_{3}$ emulsion template method can be significantly accelerated using the emulsion template of $\mathrm{PAA} / 3$, 3'-diaminodipropylamine (DDA) rather than that of PAA/ $\mathrm{NH}_{3}$. DDA acted not only as the catalyst for the sol-gel reaction, but also as a more efficient cross-linker for the PAA. Thus the emulsion templates gained much higher stability due to the PAA/DDA solution is increased viscosity. Nevertheless, replacing ammonia by other amines to increase the viscosity of the emulsion templates seems not advantageous for producing HSNPs with a small size, since alkyl groups (or other functional groups) would require an additional larger volume towards the templates. 
The present work dealt with a rapid PAA/ $\mathrm{NH}_{3}$ template method for producing HSNPs with a small size. The viscosity of the templates $\left(\mathrm{PAA} / \mathrm{NH}_{3}\right)$ is also the focus of close attention in this study. There is a positive relation between the molecular weight (MW) of the polymer and the viscosity of the polymer solution, which can be described by the Mark-Kuhn-Houwink equation [25]. The viscosity of the PAA/ $\mathrm{NH}_{3}$ solution is adjusted by the MW PAA rather than replacing ammonia with other amines. We then analyzed the effects of the MW PAA on the viscosity of the templates, $\mathrm{SiO}_{2}$ coating rate and morphology of the HSNPs, and provide explanations.

\section{Experimental section}

\subsection{Materials}

PAAs (MW=5000, 25000, 250000, Wako Pure Chemical) (MW=50000, Polysciences) were used as the emulsion templates. Tetraethoxysilane (TEOS) (Wako Pure Chemical) was used as the precursor of the silica shell. An ammonia aqueous solution $\left(\mathrm{NH}_{3}\right)(25 \%$, Wako Pure Chemical) was used as the catalyst for the sol-gel reaction and counter ion for the PAA condensation. Absolute ethanol $(99.5 \%$, Wako Pure Chemical) was used as the solvent for the sol-gel reaction. All chemicals were used as received without further purification.

\subsection{Preparation of HSNPs}

A $0.12 \mathrm{~g}$ sample of PAA was dissolved in $2.0 \mathrm{~mL}$ of a $25 \mathrm{wt} \% \mathrm{NH}_{3}$ aqueous solution with vigorous stirring lasting for $24 \mathrm{~h}$. Thereafter, $2.0 \mathrm{~mL}$ of PAA/ $\mathrm{NH}_{3}$ was added to $35.0 \mathrm{~mL}$ of ethanol and then the emulsions formed. Following that, $2.0 \mathrm{~mL}$ of TEOS was injected (aliquots totaling $1.2 \mathrm{~mL}$ at 1 hour intervals) under vigorous magnetic stirring at room temperature. At the target reaction times $(0$, $2,4,6,8,10 \mathrm{~h}$ ) after injection of the TEOS, the product was centrifuged and washed several times with distilled water. Finally, the obtained particles were dried at $180^{\circ} \mathrm{C}$ in a vacuum.

\subsection{Characterizations}

The viscosity of the PAA/ $\mathrm{NH}_{3}$ was measured by a HAAKE Rheo Stress 6000 (Thermo Fisher Scientific K.K.). The particle size of the PAA/ $\mathrm{NH}_{3}$ emulsion templates in ethanol was measured by a Zetasizer Nano (Malvern Instruments, Ltd.). The morphology of the HSNPs was determined by scanning transmission electron microscopy (STEM, JSM-7600F JEOL Ltd.). For measuring the TEOS conversion ratio, $0.1 \mathrm{ml}$ of the supernatant solution was collected by centrifugation and mixed with $20 \mathrm{ml}$ of a $5 \mathrm{M} \mathrm{NaOH}$ aqueous solution. After stirring for three days, the concentration of $\mathrm{Si}$ was measured by inductively coupled plasma atomic emission spectrophotometry (SPS7800 Seiko Instrument, Inc.). The TEOS conversion ratio was calculated using the following equation (1).

TEOS conversion ratio $(\%)=($ Initial TEOS concentration-residual silica source)/(Initial TEOS concentration $) \times 100 \cdots(1)$ 
The calibration curve was made using $0,1,10$, and $100 \mathrm{ppm}$ Si solutions. The degree of the PAA condensation in the templates was estimated by small angle x-ray scattering (SAXS, SAXSpace, Anton Paar $\mathrm{GmbH})$. A linear x-ray source of $\mathrm{CuK \alpha}$ was employed $(40 \mathrm{kV}, 50 \mathrm{~mA})$, and the collection time was set from 5 to 60 minutes to obtain a strong scattering signal at $25{ }^{\circ} \mathrm{C}$. Data processing and Guinier fitting were carried out using SAXSquant software, which is also programmed by Anton Paar.

\section{Results and Discussion}

\subsection{Effects of PAA MW on the viscosity of the $\mathrm{PAA} / \mathrm{NH}_{3}$ solution}

Table 1 shows the viscosities of the PAA/ $\mathrm{NH}_{3}$ and PAA/water using PAA with different MWs (denoted as $\mathrm{PAA}_{5000}, \mathrm{PAA}_{25000}, \mathrm{PAA}_{50000}$, and $\mathrm{PAA}_{250000}$ ). As indicated, the viscosities of the $6.0 \mathrm{wt} \%$ PAA/water solutions were $0.6,1.3,1.7$ and $53.6 \mathrm{mPa} \cdot \mathrm{s}$ when using $\mathrm{PAA}_{5,000}, \mathrm{PAA}_{25,000}, \mathrm{PAA}_{50,000}$, and $\mathrm{PAA}_{250,000}$, respectively, as shown in Table 1 (a). This tendency shows good agreement with Eq (1) that the higher MW polymer solution has a higher viscosity. The viscosities of the PAA/NH solutions were 2.1, 8.6, 14.7 and $477.0 \mathrm{mPa} \cdot \mathrm{s}$ when using $\mathrm{PAA}_{5,000}, \mathrm{PAA}_{25,000}, \mathrm{PAA}_{50,000}$, and PAA $_{250,000}$, respectively, as shown in Table 1 (b). The viscosity also increased with the increasing MW PAA in the ammonia solution. The viscosity in the ammonia solution increased more than in the water, because ammonia worked as the counter ion for the PAA condensation. Moreover, the role of ammonia became more significant when using PAA with the high MW.

\subsection{Effects of PAA MW on the morphology of the HSNPs}

Figure 1 shows STEM images of the as-synthesized HSNPs, indicating the influence of both the MW PAA and reaction time. It is hard to obtain the emulsion for $\mathrm{PAA}_{250000} / \mathrm{NH}_{3}$, since precipitation rapidly formed after those solutions were added to the ethanol due to the extremely high viscosity. When the template of $\mathrm{PAA}_{5,000} / \mathrm{NH}_{3}$ was applied (condition (a)), it seems that the time of the silica coating should be set at $4 \mathrm{~h}$ or longer for producing robust HSNPs. Otherwise, HSNPs would be obtained ((a)-0, 2h) with thin cracked shells (5-10 nm). In addition, all HSNPs samples synthesized with the $\mathrm{PAA}_{5,000} / \mathrm{NH}_{3}$ template were poly-dispersed with sizes varying from $20 \mathrm{~nm}$ to $150 \mathrm{~nm}$. When a PAA with a higher MW was used, obviously, it was much easier to obtain robust HSNPs within a short $\mathrm{SiO}_{2}$ coating time. Especially, when $\mathrm{PAA}_{50,000} / \mathrm{NH}_{3}$ emulsion templates were applied, the HSNPs could be obtained even if the silica coating was stopped at $2 \mathrm{~h}$ after the TEOS was thoroughly added in ((c)-2h).

The size of the obtained HSNPs was also significantly affected by the MW PAA. As seen in Fig. 1, HSNPs obtained using the templates of PAA ${ }_{5,000} / \mathrm{NH}_{3}, \mathrm{PAA}_{25,000} / \mathrm{NH}_{3}$ and $\mathrm{PAA}_{50,000} / \mathrm{NH}_{3}$ were 100 , 60 , and $35 \mathrm{~nm}$ in average inner size, respectively. 


\subsection{Effects of PAA MW on the size of the PAA/ $\mathrm{NH}_{3}$ template}

Figure 2 shows the size distributions curves of the $\mathrm{PAA}_{5,000} / \mathrm{NH}_{3}, \mathrm{PAA}_{25,000} / \mathrm{NH}_{3}$, and $\mathrm{PAA}_{50,000} / \mathrm{NH}_{3}$ emulsion templates in ethanol. The PAA/ $\mathrm{NH}_{3}$ emulsion templates formed in ethanol, but their sizes were different from each other. The average sizes of $\mathrm{PAA}_{5,000} / \mathrm{NH}_{3}, \mathrm{PAA}_{25,000} / \mathrm{NH}_{3}$ and $\mathrm{PAA}_{50,000} / \mathrm{NH}_{3}$ were calculated to be 210,85 , and $60 \mathrm{~nm}$, respectively. Based on Table 1, it seems that the PAA/ $\mathrm{NH}_{3}$ emulsion templates with a high viscosity were able to more easily suppress coalescence than those with a low viscosity, and thus reach a stable status with a small size. Combined with Fig. 1, the obtained HSNPs inner size was almost the same as the template size for each MW PAA. It shows that the PAA/ $\mathrm{NH}_{3}$ emulsion templates can be used as a silica coating template in ethanol.

\subsection{Effects of PAA MW on the sol-gel reaction}

The $\mathrm{SiO}_{2}$ coating rate was further analyzed by measuring the conversion ratio of TEOS. Figure 3 shows the increasing TEOS conversion ratio from $0 \mathrm{~h}$ to $10 \mathrm{~h}$. When PAA, $\mathrm{A}_{5,000} / \mathrm{NH}_{3}$ was used as the template, the conversion ratio of TEOS increased from $80 \%$ to $85 \%$ within $6 \mathrm{~h}$, and remained steady thereafter. When $\mathrm{PAA}_{25,000}$ was used as the template, the conversion ratio was $85 \%$ at $0 \mathrm{~h}$ and reached $89 \%$ at $6 \mathrm{~h}$, and further reached $91 \%$ at $10 \mathrm{~h}$. When $\mathrm{PAA}_{50,000}$ was used as the template, the conversion ratio was $93 \%$ and it reached $95 \%$ at $6 \mathrm{~h}$, then became almost constant. Obviously, the TEOS conversion ratio increased with the increasing MW PAA.

\subsection{HSNPs formation mechanism}

As already discussed, MW PAA affected both the viscosity and size of the PAA $/ \mathrm{NH}_{3}$ emulsion templates, the silica coating rate, and TEOS conversion ratio. A SAXS analysis was conducted to clarify how the MW affected this procedure. Figure 4 shows the SAXS scattering curves of the $\mathrm{PAA}_{5,000} / \mathrm{NH}_{3}, \mathrm{PAA}_{25,000} / \mathrm{NH}_{3}$, and $\mathrm{PAA}_{50,000} / \mathrm{NH}_{3}$ emulsion templates in ethanol, and the Guinier fitting curves of $\mathrm{PAA}_{25,000} / \mathrm{NH}_{3}$ and $\mathrm{PAA}_{50,000} / \mathrm{NH}_{3}$ are also shown as the solid line curves. With PAA ${ }_{5,000} / \mathrm{NH}_{3}$, the obtained result could not be calculated by Guinier fitting, because the scattering materials were too big to calculate (>100 nm). The degree of inclination of the SAXS curves shows the morphology of the scattering materials. When the scattering materials were spheres, the intensities decreased with $\mathrm{q}^{-4}$. In Fig. 4 (b, c), the scattering intensities of $\mathrm{PAA}_{25,000} / \mathrm{NH}_{3}$ and $\mathrm{PAA}_{50,000} / \mathrm{NH}_{3}$ decreased with $\mathrm{q}^{-4}$, and it showed that $\mathrm{PAA}_{25,000} / \mathrm{NH}_{3}$ and $\mathrm{PAA}_{50,000} / \mathrm{NH}_{3}$ might form stable spheres. On the other hand, in Fig. 4 (a), the scattering intensities of $\mathrm{PAA}_{5,000} / \mathrm{NH}_{3}$ did not decrease with $\mathrm{q}^{-4}$, but it showed that $\mathrm{PAA}_{5,000} / \mathrm{NH}_{3}$ might form rough spheres, because $\mathrm{PAA}_{5,000}$ had the shortest chain length in the used reagent, and $\mathrm{PAA}_{5,000} / \mathrm{NH}_{3}$ might have a higher mobility in ethanol than $\mathrm{PAA}_{25,000} / \mathrm{NH}_{3}$ and $\mathrm{PAA}_{50,000} / \mathrm{NH}_{3}$. In Fig. 4 (a, b, c), the scattering intensities were almost the same values at $0.043 \mathrm{~nm}^{-1}$. It shows that the degree of PAA condensation in the templates 
were almost the same, even if the different MW PAAs were used, and it formed different size templates.

In Fig. 4 (d, e), the pair distance distribution function (PDDF) curves of the $\mathrm{PAA}_{25,000} / \mathrm{NH}_{3}$ and $\mathrm{PAA}_{50,000} / \mathrm{NH}_{3}$ emulsion templates were calculated from the Guinier fitting curves in Fig. 4 (b, c). The PDDF curves show the distribution of the particle sizes and maximum sizes of the templates. The maximum template sizes were 70 and $50 \mathrm{~nm}$ with the $\mathrm{PAA}_{25,000} / \mathrm{NH}_{3}$ and $\mathrm{PAA}_{50,000} / \mathrm{NH}_{3}$ emulsion templates in ethanol, respectively. The curves in Fig.4 (e) were more shapely than the curve in Fig.4 (d), and it shows that it formed more uniform templates with $\mathrm{PAA}_{50,000} / \mathrm{NH}_{3}$. This result shows a similar tendency with DLS in Fig. 2.

We then proposed a mechanism for the silica coating on the PAA/ $/ \mathrm{NH}_{3}$ templates with the different MW PAAs. In this experiment, $\mathrm{NH}_{3}$ had two functions which are the counter ion for the carboxyl group of PAA and the catalyst for the sol-gel reaction. It can be found in or on the emulsion template. As seen in Figure 5, $\mathrm{NH}_{3}$ on the surface of the emulsion templates functioned as the catalyst for the sol-gel reaction, and ammonia in the emulsion templates functioned as the counter ion for the PAA condensation in the emulsion. When using the lower MW PAA, the PAA/ $\mathrm{NH}_{3}$ had a lower viscosity, thus the PAA/ $\mathrm{NH}_{3}$ formed a larger PAA/ $/ \mathrm{NH}_{3}$ template in ethanol. On the contrary, when PAA with the higher MW was used, the PAA/ $/ \mathrm{NH}_{3}$ had a higher viscosity, thus $\mathrm{PAA} / \mathrm{NH}_{3}$ formed a smaller PAA/ $\mathrm{NH}_{3}$ template in ethanol. When the emulsion templates became smaller, the template surface area also increased. For the SAXS results, the degree of the PAA condensation in the templates was the same for the different MW PAAs. This result shows that the quantity of the ammonia was same in the emulsion templates, even if the template sizes were different. As a result, when the templates had a higher surface area, the sol-gel reaction was promoted because of the increasing quantity of ammonia that could function as the catalyst for the sol-gel reaction. In addition, the emulsion templates were stabilized by the increasing MW PAA in the SAXS results. On the stable templates, the silica shell might be more easily formed than on the unstable templates (rough templates). Therefore, the TEOS conversion ratio increased, and the silica shell completely formed at 2 hours with the higher viscosity PAA/ $\mathrm{NH}_{3}$.

\section{Conclusions}

The effects of the viscosity on synthesizing HSNPs using PAA/ $\mathrm{NH}_{3}$ emulsion templates were investigated. The viscosity was changed by using four types of PAAs with the MWs of 5,000, 25,000, 50,000, and 250,000. As the results, with PAA $_{50,000}, 20 \mathrm{~nm}$ HSNPs were synthesized at 2 hours and the TEOS conversion ratio was the highest in the PAA $\mathrm{P}_{5,000}$ and $\mathrm{PAA}_{25,000}$. The PAA/NH had a higher viscosity with a higher $\mathrm{MW}$, and the PAA/ $\mathrm{NH}_{3}$ template could be stabilized with a smaller diameter. The smaller PAA/ $\mathrm{NH}_{3}$ template had a higher surface area than the larger PAA/ $/ \mathrm{NH}_{3}$ template. As a result, the TEOS could react on a larger amount of surface, and $\mathrm{NH}_{3}$ could exist on 
the surface of the PAA/NH $/ \mathrm{NH}_{3}$ template due to the increased surface area. By this mechanism, the sol-gel reaction was promoted using the higher MW PAA.

\section{Acknowledgement}

This work was supported by the Advanced Low Carbon Technology Research and Development Program (ALCA) of the Japan Science and Technology Agency (JST). The authors appreciate Mr. Koichiro Sagiyama (Anton Paar Japan K. K.) for his great support and helpful discussions in the SAXS characterizations.

\section{References}

1. M. Fuji, C. Takai, Y. Tarutani, T. Takei, M. Takahashi, Surface properties of nanosize hollow silica particle on a molecular level, Advanced Powder Technology 18 (1) (2007) 81-91.

2. J. Hu, X. Wang, L. Liu, L. Wu, A facile and general fabrication method for organic silica hollow spheres and their excellent absorption properties for heavy metal ions, Journal of Materials Chemistry A 2 (2014) 19771-19777.

3. M. Fuji, C. Takai, H. Watanabe, K. Fujimoto, Improved transparent thermal insulation using nano-spaces, Advanced Powder Technology 26 (2015) 857-860.

4. W. Suthabanditpong, R. Buntem, C. Takai, M. Fuji, T. Shirai, The quantitative effect of silica nanoparticles on optical properties of thin solid silica UV-cured films, Surface and Coatings Technology, 279 (2015) 25-31.

5. W. Suthabanditpong, C. Takai, M. Fuji, R. Buntem, T. Shirai, Studies of optical properties of UV-cured acrylate films modified with spherical silica nanoparticles, Advanced Powder Technology 27 (2016) 411-416.

6. Y. Jiao, J. Guo, S. Shen, B. Chang, Y. Zhang, X. Jiang, W. Yang, Synthesis of discrete and dispersible hollow mesoporous silica nanoparticles with tailored shell thickness for controlled drug release, Journal of Materials Chemistry, 22 (2012) 17636-17643.

7. H. Zhang, H. Xu, M. Wu, Y. Zhong, D. Wang, Z. Jiao, A soft-hard template approach towards hollow mesoporous silica nanoparticles with rough surfaces for controlled drug delivery and protein absorption, Journal of Materials Chemistry B 3 (2015) 6480-6489.

8. N. Hao, K. W. Jayawardana, X. Chen, T. D. Zoysa, M. Yan, One-step synthesis of amine-functionalized hollow mesoporous silica nanoparticles as efficient antibacterial and anticancer, ACS Applied Materials and Interfaces 7 (2) (2015) 1040-1045.

9. N. Jatupaiboon, Y. Wang, H. Wu, X. Song, Y. Song, J. Zhang, X. Ma, M. Tan, A facile microemulsion template route for producing hollow silica nanospheres as imaging agents and drug nanocarriers, Journal of Materials Chemistry B 3 (2015) 3130-3133.

10. M-W. Chang, E. Stride, M. Edirisinghe, A New Method for the Preparation of Monoporous Hollow Microspheres, Langmuir 26 (7) (2010) 5115-5121

11. K. D. Kim, K. Y. Choi, J. W. Yang, Formation of spherical hollow silica particles from sodium silicate solution by ultrasonic spray pyrolysis method, Colloids and Surfaces A: Physicochemical and Engineering Aspects, 254 (2005) 193-198

12. X. Ding, Y. Jiang, K. Yu, H. Bala, N. Tao, J. Zhao, Z. Wang, Silicon dioxide as coating on polystyrene nanoparticles in situ emulsion polymerization, Materials Letters 58 (2004) $1722-1725$.

13. X. Ding, K. Yu, Y. Jiang, H. Bala, H. Zhang, Z. Wang, A novel approach to the synthesis of hollow silica nanoparticles, Materials Letters 58 (2004) 3618-3621.

14. C. Takai, T. Ishino, M. Fuji, T. Shirai, Rapid and high yield synthesis of hollow silica nanoparticles using an $\mathrm{NH}_{4} \mathrm{~F}$ catalyst, Colloids and Surfaces A: Physicochemical and Engineering Aspects 446 (2014) 46-49 
15. Z-Z. Li, S-A. Xu, L-X. Wen, F. Liu, A-Q. Liu, Q. Wang, H-Y. Sun, W. Yu, J-F. Chen, Controlled release of avermectin from porous hollow silica nanoparticles: Influence of shell thickness on loading efficiency, UV-shielding property and release, Journal of Controlled Release 111 (2006) 81-88.

16. Y. Le, J-F. Chen, J-X. Wang, L. Shao, W-C. Wang, A novel pathway for synthesis of silica hollow spheres with mesostructured walls, Materials Letters 58 (2004) 2105-2108.

17. Y. Wan, S-H. Yu, Polyelectrolyte controlled large-scale synthesis of hollow silica spheres with tunable sizes and wall thickness, The Journal of Physical Chemistry C 112 (2008) 3641-3647.

18. L. M. Liz-Marzan, M. Giersig, P. Mulvaney, Synthesis of nanosized gold-silica core-shell particles, Langmuir 12 (1996) 4329-4335.

19. X. Du, L. Yao, J. He, One-pot fabrication of noble-metal nanoparticles that are encapsulated in hollow silica nanospheres: dual roles of poly (acrylic acid), Chemistry A European Journal 18 (2012) 7878-7885.

20. R. V. R. Virtudazo, R. T. Wu, S. Zhao, M. M. Koebel, Facile ambient temperature synthesis and characterization of a stable nano-sized hollow silica particles using soluble-poly (methacrylic acid) sodium salt templating, Materials Letters 126 (2014) 92-96

21. M. Fuji, C. Takai, H. Imabeppu, X. Xu, Synthesis and shell structure design of hollow silica nanoparticles using polyelectrolyte as template, Journal of Physics: Conference Series 596 (2015) 012007.

22. Y. Shi, C. Takai, T. Shirai, M. Fuji, Facile synthesis of hollow silica nanospheres employing anionic PMANa templates, Journal of Nanoparticle Research 17 (2015) 204-213

23. Y. Du, L. E. Luna, W. S. Tan, M. F. Rubner, R. E. Cohen, Hollow silica nanoparticles in UV-Visible antireflection coatings for poly (methyl methacrylate) substrates, ACS Nano 4 (7) (2010) 4308-4316.

24. C. Takai-Yamashita, H. Imabeppu, M. Fuji, Synthesis of hollow silica nanoparticles using poly (acrylic acid)-3,3'-diaminodipropylamine template, Colloids and Surfaces A: Physicochemical and Engineering Aspects 483 (2015) 81-86.

25. H. L. Wagner, The Mark-Houwink-Sakurada Equation for the viscosity of linear polyethylene, Journal of Physical and Chemical Reference Data 14 (2) (1985) 611-617 
Table 1 . Viscosity of PAA aqueous and PAA/ $\mathrm{NH}_{3}$ for each MW PAA

(a) $6 \mathrm{wt} \%$ PAA aq. (b) $\mathrm{PAA} / \mathrm{NH}_{3}$

\begin{tabular}{cccc} 
& MW PAA & \multicolumn{2}{c}{ Viscosity / mPa $\cdot \mathrm{s}$} \\
\cline { 3 - 4 } PAA $_{5,000}$ & 5,000 & 0.6 & 2.1 \\
PAA $_{25,000}$ & 25,000 & 1.3 & 8.6 \\
PAA $_{50,000}$ & 50,000 & 1.7 & 14.7 \\
PAA $_{250,000}$ & 250,000 & 53.6 & 477.0 \\
\hline
\end{tabular}




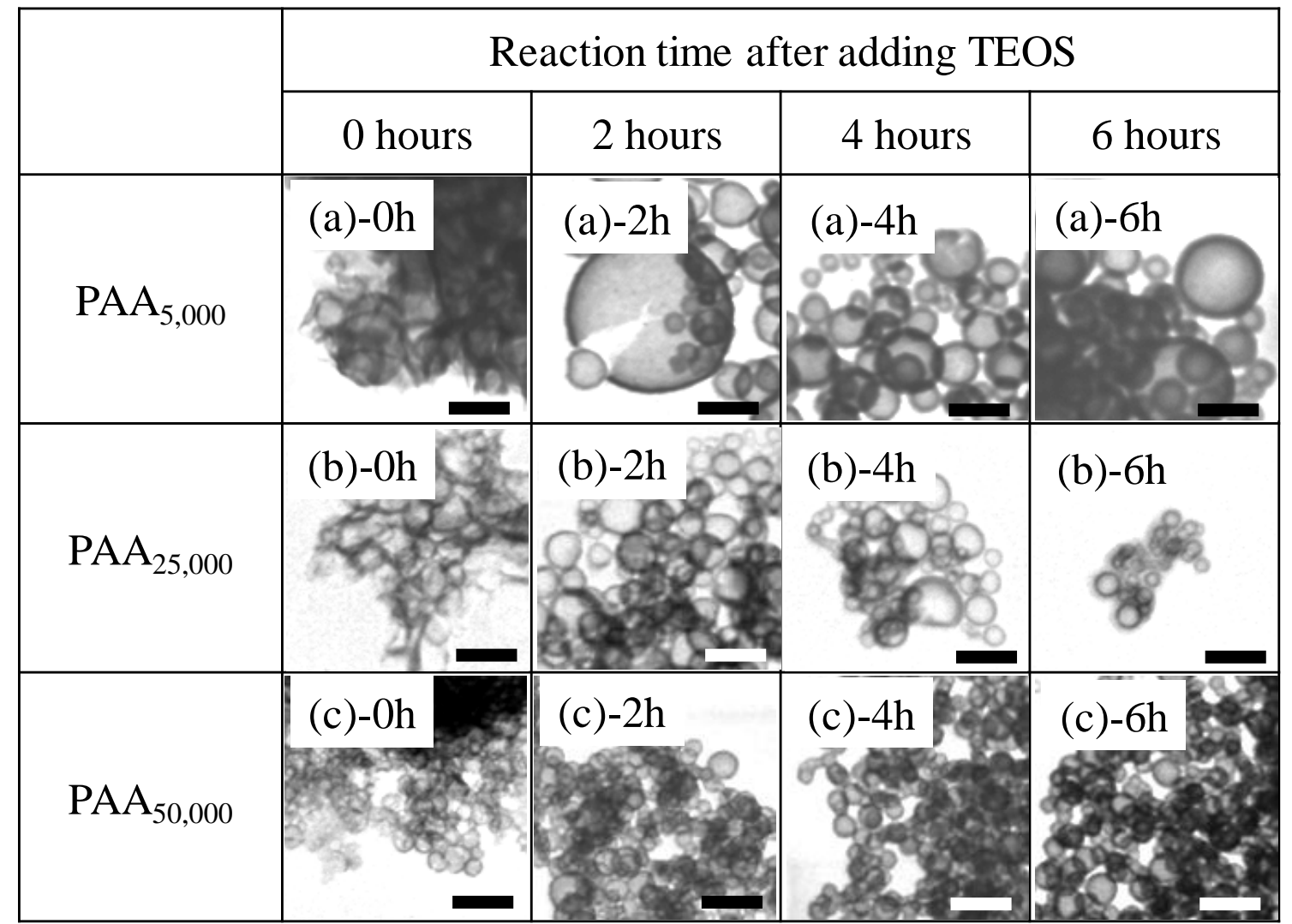

Fig. 1. STEM images of HSNPs prepared using (a) $\mathrm{PAA}_{5,000} / \mathrm{NH}_{3}$, (b) $\mathrm{PAA}_{25,000} / \mathrm{NH}_{3}$, and (c) $\mathrm{PAA}_{50,000} / \mathrm{NH}_{3}$ templates. All the scale bars represent $100 \mathrm{~nm}$. 


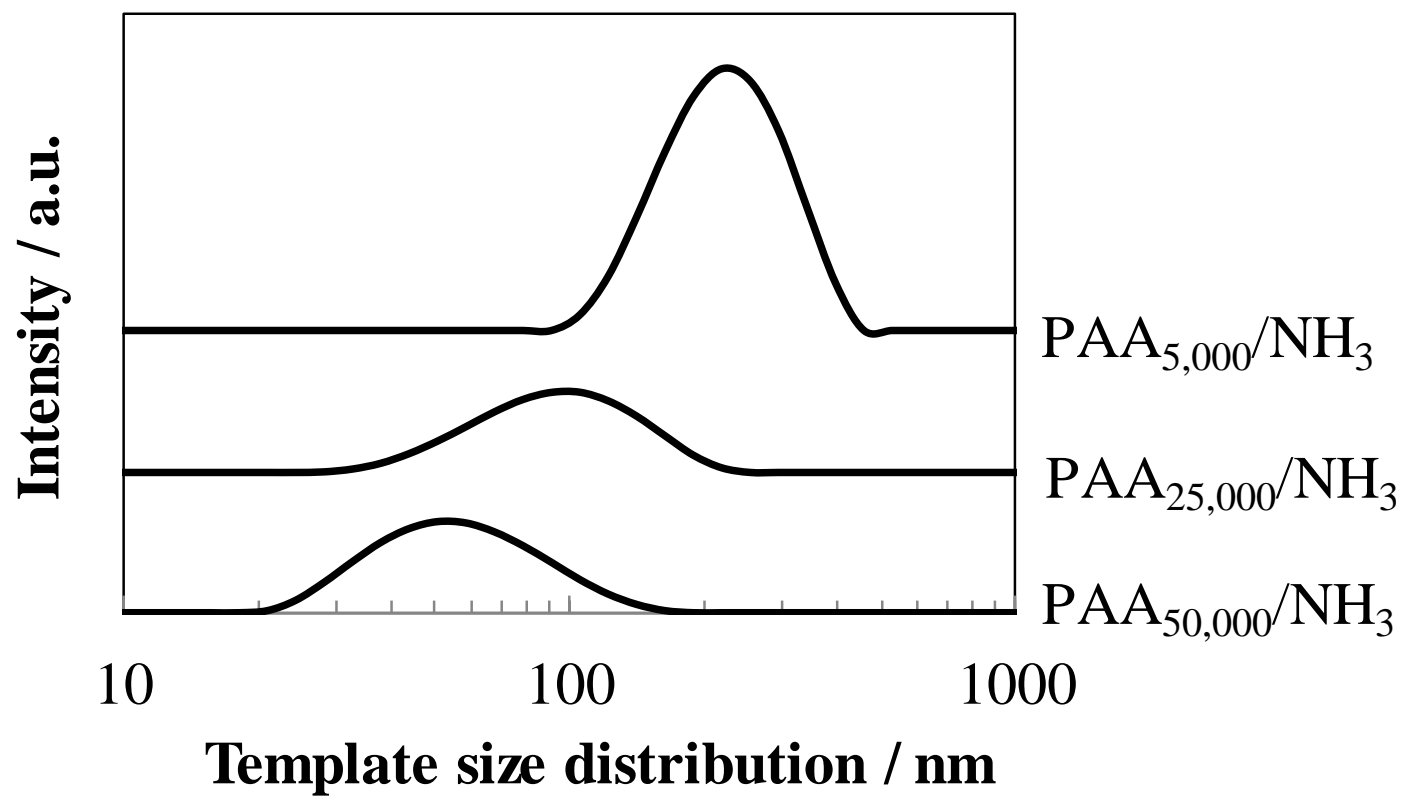

Fig. 2. Emulsion template size distributions of $\mathrm{PAA} / \mathrm{NH}_{3}$ for each $\mathrm{MW}$ PAA in ethanol 


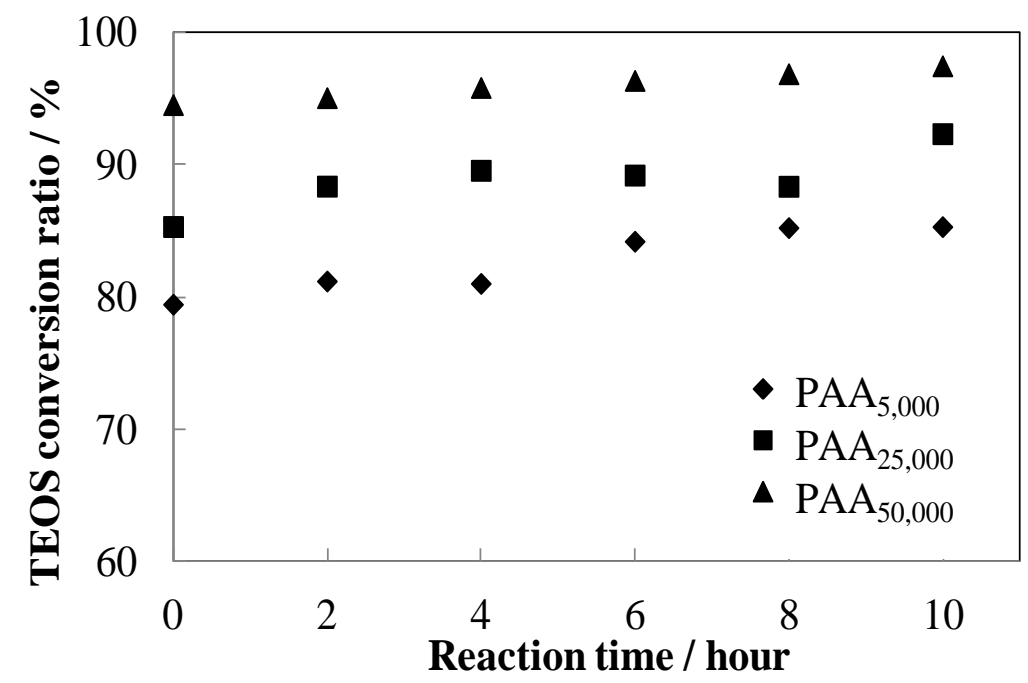

Fig. 3. TEOS conversion ratio from $0 \mathrm{~h}$ to $10 \mathrm{~h}$ after TEOS addition using PAA $\mathrm{A}_{5,000}$, $\mathrm{PAA}_{25,000}$ and PAA 50,000 as the template polymers 

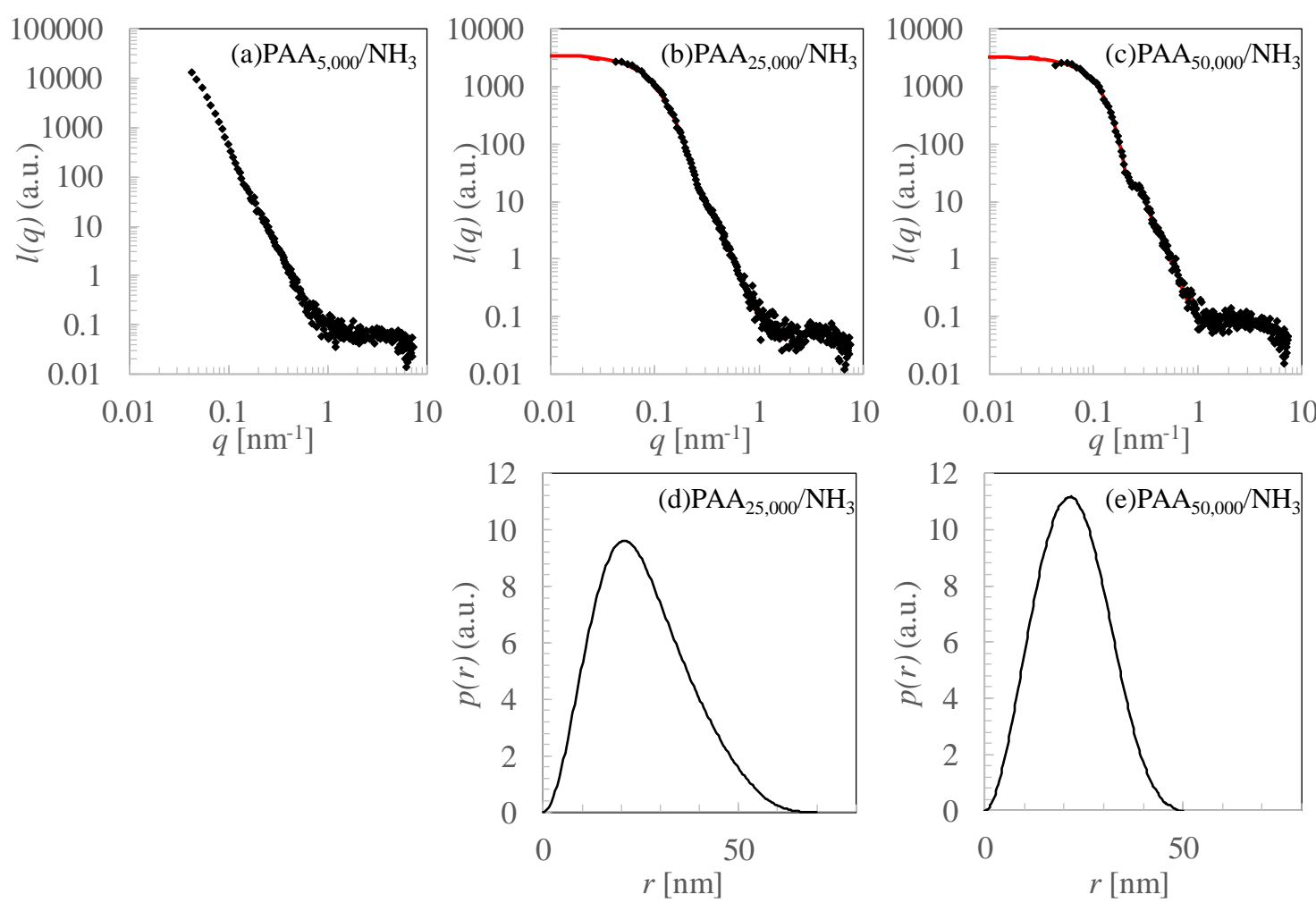

Fig. 4. SAXS curves and fitting curves of PAA/NH $\mathrm{NH}_{3}$ templates using (a)PAA $\mathrm{P}_{5,000}$, (b) $\mathrm{PAA}_{25,000}$, and (c) $\mathrm{PAA}_{50,000}$. The calculated PDDF curves of the PAA/ $\mathrm{NH}_{3}$ templates using (d)PAA ${ }_{25,000}$, and (e) $\mathrm{PAA}_{50,000}$. 

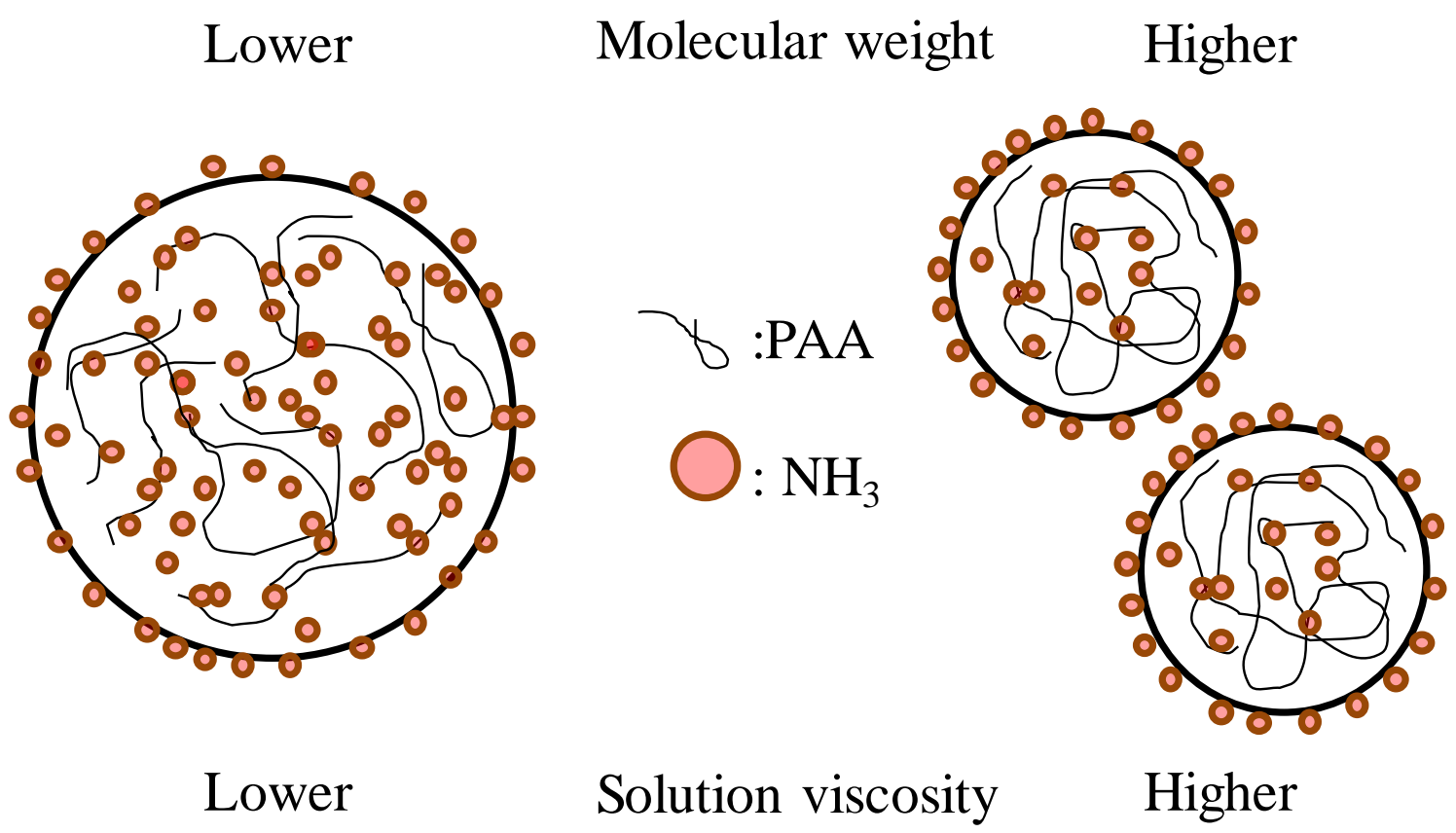

Figure. 5. Images of $\mathrm{PAA} / \mathrm{NH}_{3}$ templates with higher and lower MWs of PAAs 
Template Viscosity

High

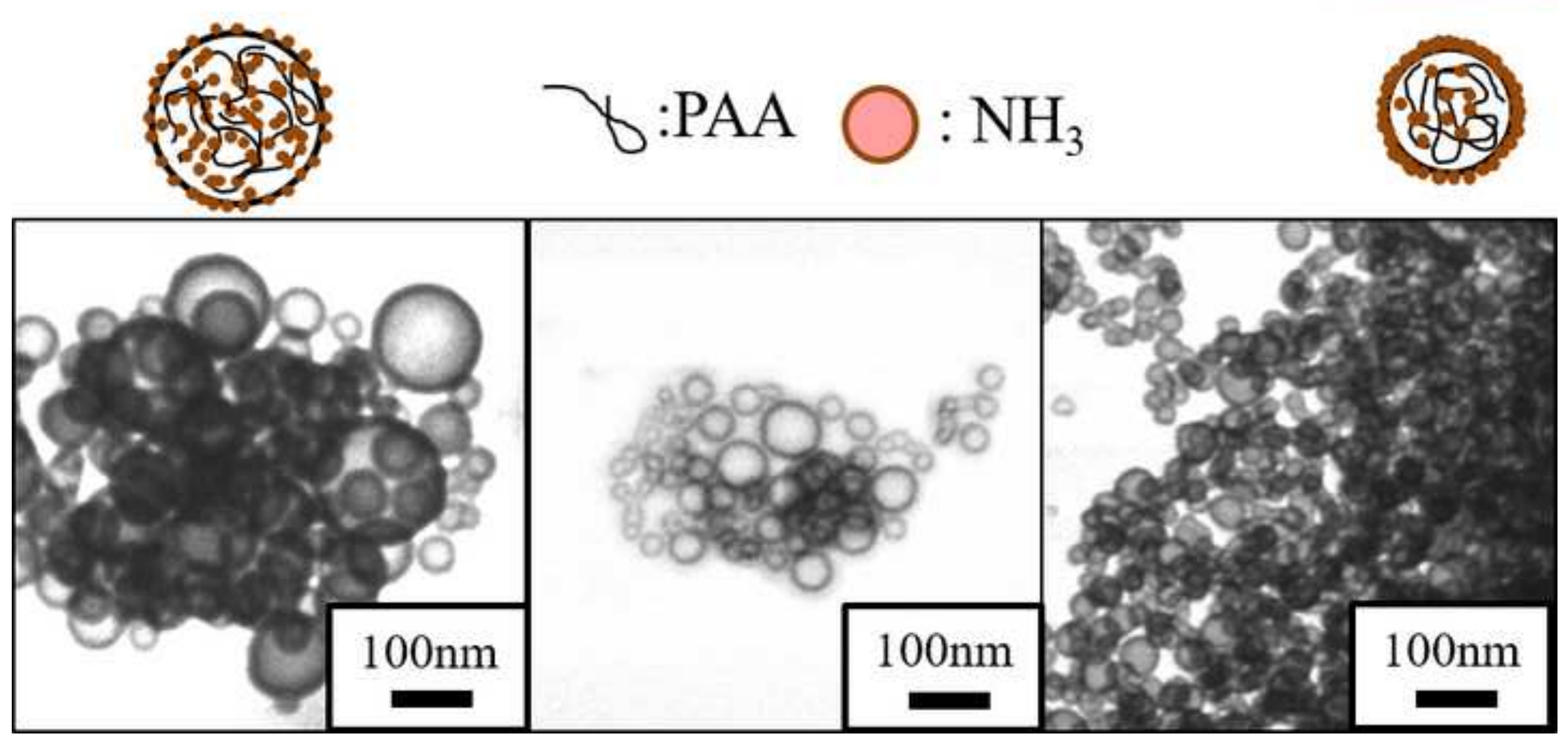

